\title{
Condiciones adversas para la ciencia al nacer la Universidad Nacional de México
}

\author{
María de la Paz Ramos-Lara
}

\begin{abstract}
RESUMEN
En México, a diferencia de otros países, la mayor parte de la investigación científica de frontera se realiza en las universidades públicas. Este vínculo inició en 1910 con la creación de la Universidad Nacional de México, entre cuyos objetivos se encontraba el desarrollo de la ciencia básica. Utilizando un enfoque sistémico, este trabajo muestra que esa misión, inicialmente, era inviable por la desmedida apertura que se otorgó a los inversionistas extranjeros durante el porfiriato (1877-1911). La Revolución mexicana combatió esa estructura económica y fue la autonomía universitaria (1929) la que legalmente concedió libertad para desarrollar ciencia de frontera, dando lugar a la institucionalización de la investigación científica correspondiente y a la profesionalización de las ciencias físico-matemáticas.
\end{abstract}

Palabras clave: UNAM; ciencia, tecnología y sociedad; ciencia y desarrollo; autonomía universitaria; educación y desarrollo; México.

\section{Condições adversas para a ciência ao nascer a Universidad Nacional de México}

\section{RESUMO}

No México, a diferença de outros países, a maior parte da pesquisa científica de fronteira se realiza nas universidades públicas. Este vínculo iniciou-se em 1910 com a criação da Universidad Nacional de México, quando um dos objetivos era o desenvolvimento da ciência básica. Utilizando um enfoque sistémico, este trabalho mostra que essa missão, inicialmente, era inviável pela desmedida abertura que se deu aos investidores estrangeiros durante o porfiriato (1877-1911). A Revolução mexicana combateu essa estrutura económica e foi a autonomia universitária (1929) que legalmente concedeu liberdade para desenvolver ciência de fronteira, dando lugar à institucionalização da pesquisa científica correspondente e à profissionalização das ciências físico-matemáticas.

Palavras chave: Universidad Nacional Autónoma de México (UNAM); ciência, tecnologia e sociedade; ciência e desenvolvimento; autonomia universitária; educação universitária, México.

\section{María de la Paz Ramos-Lara}




\section{Adverse Conditions for Science during the First Years of the National University of Mexico}

\section{ABSTRACT}

In Mexico, unlike other countries, most frontier scientific research is carried out in public universities. This link began in 1910 with the creation of the National University of Mexico, whose objectives included the development of basic science. Using a systemic approach, this work shows that this mission was initially unfeasible due to the excessive opening granted to foreign investors during the Porfiriato (1877-1911). The Mexican Revolution fought off this economic structure and it was university autonomy (1929) that legally granted freedom to develop frontier science, leading to the institutionalization of the corresponding scientific research and the professionalization of the physical-mathematical sciences.

Key words: National Autonomous University of Mexico (UNAM); science, technology and society; science and development; university autonomy; university education, Mexico. 


\section{Introducción}

En este ensayo analizo, mediante un enfoque sistémico, la relación que hubo entre la educación superior pública y las ciencias exactas, así como su interacción con el entorno socioeconómico desde el comienzo del régimen de Porfirio Díaz (1877) — promotor de la creación de la Universidad Nacional de México (UNM), en $1910{ }^{1}{ }^{1}$ hasta que esta Universidad adquirió su autonomía universitaria (1929). ${ }^{2}$ En estos términos, nuestro sistema está integrado por la universidad pionera, la UNM, y la institución de educación superior que en ese periodo tenía el liderazgo en el desarrollo de la ciencias exactas - la física, las matemáticas y la química-, la Escuela Nacional de Ingenieros (ENI). De manera tangencial se considera a la Escuela Nacional de Medicina (ENM) pues sus miembros y egresados, al igual que los de la ENI, conformaban una comunidad científico-académica sólida y reconocida en el país.

Por el otro lado está el medio externo, delimitado por las condiciones económicas, políticas y culturales del país en el periodo señalado. En la interacción de los elementos del sistema (las instituciones de educación superior) con el medio externo (el aparato gubernamental), se produjeron diferencias y conflictos que obstaculizaron el desarrollo de la ciencia hasta que, en 1929, la autonomía universitaria separó los intereses de ambos sectores, ofreciendo a la comunidad académica un marco jurídico apropiado para desarrollar libremente la ciencia según sus inquietudes particulares, entre ellas, la ciencia de frontera.

A continuación expongo brevemente el enfoque sistémico utilizado.

\section{Una visión sistémica}

Para estudiar la historia, varios autores han señalado la importancia de utilizar enfoques sistémicos. Entre ellos se encuentra el padre de la teoría general de sistemas, Karl Ludwig von Bertalanffy, e incluso el premio nobel en Química, Ilya Prigogine. La justificación del filósofo austriaco radica en que "conceptos, modelos y leyes parecidos surgen una y otra vez en campos muy diversos" (Bertalanffy, 1968: 33).

En particular, la teoría de sistemas complejos se ha introducido exitosamente en una amplia gama de campos del conocimiento -incluyendo las ciencias sociales - debido a que hay sistemas complejos en todos los ámbitos de la naturaleza, la vida y la sociedad. Al presentar la peculiaridad de compartir características en común, éstos hacen factible la elaboración de modelos aplicables en diversas áreas del conocimiento (Martínez, 2012).

Este enfoque examina las interacciones entre los elementos que integran el sistema, con el fin de buscar su dinámica y deducir sus estructuras (P. Miramontes, 1999 a: 77). Además y debido a que los sistemas complejos presentan propiedades genéricas, se hace posible identificar propiedades dinámicas similares entre sistemas cuyos componentes pueden ser muy distintos, como el sistema nervioso, la tectónica de placas, el crecimiento urbano y las economías de mercado, por mencionar algunos ejemplos (O. Miramontes, 1999 b: 84-85).

Rolando García define un sistema complejo como "una representación de un recorte de la realidad, conceptualizado como una totalidad organizada, en la cual los elementos no son 'separables' y, por tanto, no pueden ser estudiados aisladamente" (García,

\footnotetext{
${ }^{1}$ Durante el Porfiriato (1877-1911), México experimentó estabilidad social y política, además de un importante desarrollo económico, aunque el beneficio fuera para pocos, en su mayoría empresarios extranjeros y familias acomodadas simpatizantes con el gobierno del mandatario Porfirio Díaz. Gracias a la estabilidad social y a las garantías que otorgaba su gobierno para instalar empresas, la inversión extranjera se estableció con facilidad y con el paso de los años se incrementó considerablemente, en parte debido a que el marco jurídico les otorgaba facilidades y les garantizaba el pago de las inversiones. Los empresarios que predominaron fueron estadounidenses, ingleses, franceses y alemanes, entre otros.

${ }^{2}$ Con la autonomía, la UNM cambió su denominación a la actual, Universidad Nacional Autónoma de México (UNAM). Conviene aclarar que las fuentes primarias de consulta general para esta investigación, fueron: Archivo General de la Nación; Archivo Histórico de la Universidad Nacional Autónoma de México(AHUNAM), Fondos: Escuela Nacional de Ingenieros, Escuela Nacional de Altos Estudios y Escuela Nacional de Ciencias Químicas; Archivo Histórico del Palacio de Minería de la Facultad de Ingeniería.
} 
2006: 21). El término de estructura considera el concepto dinámico que incorpora la variación y el cambio, pues la evolución de un sistema abierto se lleva a cabo mediante reestructuraciones y desestructuraciones, las cuales pueden tener lugar por interacción de los componentes internos y también con los del medio externo (García, 1993: 102-109). ${ }^{3}$

Las interacciones entre el sistema y el medio externo pueden generar fluctuaciones de pequeña escala produciendo cambios sin alterar las relaciones fundamentales que definen la estructura del sistema. Si las fluctuaciones son mayores y exceden un cierto umbral, provocarán una desorganización de la estructura. En relación con el último caso, García estudió la evolución de las sequías recurrentes en el Sahel africano y mostró que las más catastróficas no fueron producto de un recrudecimiento en las condiciones climáticas, sino que la responsabilidad total del desequilibrio se debió a la vulnerabilidad del sistema causada por factores socioeconómicos (García, 1993: 107-108).

Desde esta visión, considero que las interacciones entre los elementos que se conjugaban en el periodo histórico que analizo, nos permiten interpretar el camino que llevó a la institucionalización de la investigación científica y a la profesionalización de las ciencias físico-matemáticas en México.

\section{Inicios de la educación científico-técnica}

En los últimos años del periodo virreinal en México coexistieron varias instituciones de educación superior, de las cuales tres (precursoras en el continente americano cada una en sus ámbitos) son relevantes para este trabajo. La Real y Pontificia Universidad - creada por Real Provisión el 21 de septiembre de 1551 y abierta en 1553- que ofreció los mismos estudios y grados que las universidades europeas (Méndez 1990); el Real Seminario de Minería, fundado en 1792 y patrocinado por el Real Tribunal de Minería para formar expertos en minas que mejoraran la producción del sector minero aplicando conocimientos de ciencia moderna. ${ }^{4}$ La Real Academia de San Carlos de las Nobles Artes de Nueva España, la primera escuela de grabado abierta en 1781. Estas dos últimas integradas a la Universidad Nacional de México (UNM) en 1910.

Las tres tuvieron una vida azarosa durante el siglo XIX, una vez que México adquirió su Independencia de la metrópoli ibérica en 1821. Cosío Villegas resume la situación política del país entre 1810 y 1867 de la siguiente manera:

lucha interminable de las 'facciones': monárquicos contra republicanos, centralistas contra federalistas, conservadores contra liberales, liberales puros contra moderados, juaristas contra lerdistas y porfiristas, lerdistas contra porfiristas e iglesistas, etc. Y por si algo faltara para remachar el carácter disgregador de la época, sobrevienen cuatro guerras extranjeras, siempre humillantes, y una de las cuales le cuesta al país la pérdida del territorio (Cosío, 1963: 76-77).

En este sombrío escenario, la Real Universidad de México fue clausurada en 1833, en 1857, en 1861 y de manera definitiva en 1867, por el emperador Maximiliano de Habsburgo. ${ }^{5}$

\footnotetext{
${ }^{3}$ Las "condiciones de contorno" están vinculadas a los flujos de intercambio del sistema con el exterior, los cuales pueden ser flujos de energía, de materia o de información, por mencionar algunos ejemplos. Estos flujos pueden producir retroalimentación positiva, o bien, negativa.

${ }^{4}$ De acuerdo con su primer director, Joaquín Velázquez Cárdenas de León, los campos fundamentales para cumplir su cometido eran las matemáticas, la física experimental, la química, la mineralogía y la metalurgia. En matemáticas se estudiaría aritmética, geometría, trigonometría y álgebra. En física experimental los temas propuestos fueron: mecánica, maquinaria, hidrostática, hidráulica, aerometría y pirotecnia. En relación con química, se incorporó tanto la teórica como la práctica (Lassaga y Velázquez, 1774: 36-37).

${ }^{5}$ El emperador austriaco se interesó en el Colegio de Minería, visualizándolo como Escuela Imperial de Minas e, incluso, Escuela Politécnica, sin obtener éxito en sus pretensiones, en parte por la renuencia del personal académico de perder su estatus y tradición como escuela de minas.
} 
El Real Seminario de Minería, fundado cuando Nueva España sobresalía como líder mundial en la producción de plata, tuvo una trayectoria diferente por la formación científica que ofrecía, ya que la ciencia era reconocida mundialmente como fundamental en la modernización de cualquier sector productivo, no sólo el de la minería. Esto se sumaba al hecho de que el director fundador, el afamado mineralogista español Fausto Delhuyar, egresado de la Academia de Minas de Freiberg, la más reputada en esa época, había posicionado a la escuela de minas novohispana entre las ocho mejores del mundo y la primera en funcionar exitosamente en el Continente Americano, con un nivel superior al de la metrópoli, la Academia de Minas de Almadén.

Por otra parte, el esplendor económico de la minería novohispana de esos tiempos llevó a que se le construyera un majestuoso edificio a la escuela de minas de México, el Palacio de Minería. En sus últimos años, Nueva España contaba con los elementos requeridos para promover tanto el desarrollo de la ciencia como la innovación tecnológica. La riqueza económica era uno de ellos y, otra, una excelente formación científica de sus miembros. Esta última ya la tenían los ilustrados novohispanos, entre ellos el brillante matemático y astrónomo Velázquez de León, quien propuso la creación de este colegio metálico.

Sin embargo Delhuyar, como director fundador, decidió contratar a profesores europeos y en la medida de sus posibilidades que fueran egresados de la academia alemana, como fue el caso del notable químico y mineralogista Andrés Manuel del Río, quien reportó la primera contribución al conocimiento universal desde el territorio novohispano, el descubrimiento de un elemento (el vanadio) en 1801. La conjunción de estos elementos, entre otros, propició la innovación tecnológica, especialmente en la construcción de máquinas que funcionaran de acuerdo con las características propias de las regiones locales. Esta estrategia resultó más efectiva que los infructuosos esfuerzos por adaptar las máquinas traídas del viejo continente y diseñadas para otro contexto geográfico.

El auge del Real Seminario de Minería, al igual que el de la Real Academia de San Carlos y la Real Universidad de México, empezó su descenso con los primeros brotes de violencia que buscaban la emancipación de México de España, con lo cual la producción minera decayó rápidamente. Con la declaración de Independencia del país, la educación superior inició su reestructuración aun a pesar de las diferencias entre aquellos que pretendían mantener las instituciones virreinales, y los que preferían modernizar la educación, especialmente aquella basada en el conocimiento científico.

Las tres instituciones se vieron afectadas por la crisis social, política y económica del país durante los dos primeros tercios del siglo XIX. La Real Universidad de México fue clausurada de manera definitiva y el también denominado Colegio de Minería, al igual que la Real Academia de San Carlos, empezaron a padecer la crisis económica interna y las invasiones extranjeras que paralizaron temporalmente sus actividades. A pesar de esta sombría situación, el Colegio de Minería fue considerado útil en la modernización del país sin importar la inclinación política del grupo en el poder, por ello se incorporó al sector gubernamental algunos años después de la Independencia de México.

El hecho de que esta institución pionera y cardinal en la enseñanza científico-técnica se incorporara a la estructura oficial trazó el camino que seguiría la ciencia hasta el siglo XX, el mismo que tomaría la UNM, proyecto universitario al cual se integró, como ENI, al igual que la Escuela Nacional de Bellas Artes (ENBA), antes Academia de San Carlos. El Colegio de Minería se transformó varias veces durante el siglo XIX de acuerdo con los intereses del grupo en el poder; en las dos primeras conservó su esencia de escuela de minas, cuando se le denominó Tercer Establecimiento de Ciencias Físicas y Matemáticas, en 1833, e Instituto de Ciencias Naturales en 1843. En 
1867 se convirtió en Escuela Especial de Ingenieros y en 1883 en ENI, la principal en el país en formación de ingenieros.

\section{Ciencias físicas e ingeniería durante el porfiriato}

En México, en el último tercio del siglo XIX era evidente que el sistema educativo se perfilaba como una plataforma primordial para el desarrollo de la ciencia, dada la marginación que recibía el sector industrial nacional. Con estas limitaciones, la comunidad científico-académica con apoyo del Ministerio de Instrucción Pública promovió que las materias de física, matemáticas, química y biología se consideraran fundamentales en la enseñanza primaria, secundaria y superior. ${ }^{6}$ Se reconocía que la física, las matemáticas y la química, entre otros campos, estaban estrechamente asociados a la modernización del país. Bastaba con ver los asombrosos avances y las sorprendentes aplicaciones que éstas tenían en los países industrializados y que llegaban hasta la sociedad mexicana. Por ejemplo, la modernización en los medios de comunicación (telégrafo y teléfono), en los transportes (en ferrocarriles, transporte eléctrico y de motor) y en la iluminación eléctrica, por mencionar algunas aplicaciones de la física.

Es así como se estimulan y retroalimentan los lazos de colaboración entre la comunidad científicoacadémica mexicana y el sector gubernamental, esto es, para modernizar los programas y planes de estudio en el conocimiento científico moderno no sólo en el nivel superior, sino también en el medio y el primario. En lo que se refiere al nivel medio, la Escuela Nacional Preparatoria $(\mathrm{ENP})^{7}$ sobresalió como la primordial. Ésta se creó en 1867 bajo una concepción positivista y con una interacción muy estrecha con la ENI, la ENM, la ENBA y la Escuela Nacional de Jurisprudencia (ENJ) ${ }^{8}$ es decir, con las escuelas nacionales. Este proyecto educativo preparatoriano llegó a ser tan eficiente (por la retroalimentación positiva entre los elementos del sistema) que se posicionó como el primer programa educativo sistémico exitoso en México, con el cual se fundó la Máxima Casa de Estudios del país en 1910, la UNM (Ramos-Lara, 2018).

No es de extrañar que el sistema preparatoriano se haya considerado el mejor cimiento para construir el sistema universitario más trascendental de la nación mexicana, pues mantenía retroalimentación con la ENI, la institución de educación superior de mayor nivel y prestigio en las ciencias exactas; con la ENM que lo era en las ciencias químicas y naturales (o biológicas), y lo mismo sucedió con la ENBA en arquitectura y artes.

Los miembros y egresados de esas instituciones fueron y seguían siendo los fundadores e integrantes de las sociedades y las instituciones científicas más reconocidas en la nación. Entre ellas podemos mencionar al Observatorio Astronómico Nacional (1863), el Instituto Geológico Nacional (IGN) (1888) y el Observatorio Meteorológico (1877), entre otros. ${ }^{9}$ Con relación a las sociedades científicas, en 1884 se funda la Sociedad Científica Antonio Alzate, con una amplia red de vínculos tanto con la comunidad científica mexicana como con la internacional, que derivó en la Academia Nacional de Ciencias en 1930 (Gallardo et al., 2005); la Sociedad Mexicana de Geografía y Estadística (1833); la Sociedad Científica "Alejandro Humboldt" (1861); la Asociación de Ingenieros Civiles y Arquitectos (1868); la Academia de Ciencias Exactas, Físicas y Naturales; la Sociedad Geológica (1904), etcétera. Entre las

\footnotetext{
${ }^{6}$ En esa época esos eran los tres niveles de estudios, y el secundario coincidía con el preparatorio.

${ }^{7}$ La ENP, vinculada a programas de estudios profesionales, se creó en 1867 bajo el régimen juarista con la denominación de Escuela Preparatoria.

${ }^{8}$ La ENJ se creó al mismo tiempo que la ENP, y poco a poco sus profesores retroalimentaron directamente a la ENP e incidieron en ella principalmente en las humanidades.

${ }^{9}$ Entidades activas en la UNAM todavía, el IGN como Instituto de Geología.
} 
publicaciones periódicas que fundaron, se encuentran: Memorias de la Sociedad Científica "Antonio Alzate", el Anuario del Observatorio Astronómico Nacional, el Boletín de la Sociedad Mexicana de Geografía y Estadística, los Anales de la Comisión Geológica, y el Boletín del Instituto Geológico, por mencionar algunas (De Gortari, 1980: 316-317).

De acuerdo con este contexto, se comprende el papel decisivo que jugaron los egresados de la ENI, al igual que los de la ENM, en la profesionalización e institucionalización de la ciencia, contribuyendo a que su escuela (además de transitar institucionalmente de Real Seminario de Minería a ENI y finalmente a Facultad de Ingeniería de la UNAM) mereciera la denominación de La Primera Casa de las Ciencias en México, piedra angular en la creación de entidades pioneras en sus campos, como la Facultad de Ciencias (1938), el Instituto de Matemáticas (1942), el Instituto de Física (1938), el Instituto de Geofísica (1949), el Instituto de Geografía (1933), el Instituto de Geología (1929) y el Instituto de Química (1941) de la UNAM, entre otros (Izquierdo, 1958).

Con esta información pretendo hacer evidente que, durante el siglo XIX, la ENI tenía la solidez para atender los proyectos gubernamentales de modernización del país e, incluso, para resolver los problemas de la industria nacional. Sin embargo, su campo de acción laboral se vio severamente limitado y dañado por la llegada de empresas extranjeras que imponían sus reglas laborales y de operación, además de contratar arbitrariamente a los ingenieros extranjeros. Esto provocó que las carreras de ingeniería de la ENI asociadas a la industria entraran en franca decadencia (Ramos-Lara, 2013), dejando a la ingeniería civil como la más importante, en la ENI y a nivel nacional, hasta mediados del siglo XX, debido a su demanda en la construcción de obras públicas y de infraestructura (Domínguez, 2013).

\section{Contrariedades de los estudios de ingeniería eléctrica e industrial}

En el mundo, a finales del siglo XIX, los avances de la física, la química y las matemáticas se convirtieron en la plataforma de lanzamiento del sector industrial moderno, situación que precisó la creación de renovadoras profesiones requeridas para satisfacer las expectativas que demandaba el amplio campo laboral, como la ingeniería industrial, la ingeniería eléctrica y la ingeniería mecánica, por mencionar algunas.

En ese periodo se formaron grandes empresas trasnacionales que se instalaron en diversas partes del mundo y que empleaban un gran número de ingenieros, que en su mayoría habían sido formados en sus naciones, o en otros países industrializados. Cuando algunas de estas corporaciones se empezaron a instalar en México en el último tercio del siglo XIX, las industrias mexicanas perdieron su capacidad de competir con enormes corporaciones, de magnos capitales y aliados con la élite en el poder. Esa situación perjudicó también al sector educativo, dado que los empresarios extranjeros no solían contratar profesionales mexicanos ni formados en México. Fue una situación que afectó hondamente aquellos programas de estudios creados con el objetivo de formar personal capacitado para intervenir en el sector industrial, entre ellos los ingenieros, como se mostrará más adelante.

A su vez, este contexto económico, político y laboral (factores externos), absolutamente desfavorable para los sectores laboral y educativo, hizo imposible la creación de nuevas especialidades de ingeniería vinculadas a la industria, como la carrera de Ingeniería Química, cuyo plan de estudios fue propuesto por catedráticos de la ENI en 1911, sin posibilidades de cristalizar en ese momento. En 1918 surgió nuevamente la idea pero ahora en la Facultad de Ciencias Químicas de la UNM, pero los estragos de la Revolución mexicana también lo impidieron. Fue hasta 1927 que fructificó. ${ }^{10}$

\footnotetext{
${ }^{10}$ El plan de estudios de la carrera de Ingeniero Químico incluía las siguientes materias: Legislación Industrial y Economía Política; Higiene de Establecimientos Industriales (Bacteriología); Elementos de Mineralogía, Geología y Metalurgia; Electro-Química, Química Analítica, Química Industrial y Química-Física (Ramos-Lara, 2014).
} 


\section{Declive de las carreras relacionadas con la industria}

Durante el siglo XIX, los programas educativos de las escuelas nacionales asociados a la industria se concentraban en dos escuelas principalmente: la ENI y la ENM. En la primera se promovían carreras como Ingeniero Electricista, Ingeniero Industrial e Ingeniero Mecánico, en tanto que en la segunda era importante la de Farmacéutico. En todas ellas los perjuicios fueron considerables, pues las compañías foráneas contrataban del extranjero a su personal especializado y técnico, ${ }^{11}$ y muchas veces hasta los mismos empleados y obreros. En la ENI, esta situación incidió negativamente en las profesiones que se habían creado durante el siglo XIX (ver cuadro 1).

En el cuadro 1 se observa que, por ser una escuela de minas, de 1792 a 1866, las carreras estaban asociadas a la minería, a la agrimensura y a la geografía. A partir de su transformación en Escuela Especial (luego Nacional) de Ingenieros se incorporaron otras especialidades como la Ingeniería Civil y la Ingeniería Mecánica, y años más tarde la Ingeniería Eléctrica. Para la década de los noventa del siglo XIX era evidente la decadencia de las carreras asociadas a la industria, como se muestra en la gráfica 1. Poco más o menos a la mitad de la gestión porfiriana, cuando la economía nacional dependía de las inversiones extranjeras principalmente.

\section{Cuadro 1. Denominaciones e incremento en las carreras de ingeniería en la ENI durante el siglo XIX}

\begin{tabular}{|c|c|c|c|}
\hline 1792 & 1833 & 1861 & 1892 \\
\hline Real Seminario de Minería & $\begin{array}{c}\text { Establecimiento de Ciencias Físicas } \\
\text { y Matemáticas }\end{array}$ & Colegio Nacional de Minería & Escuela Nacional de Ingenieros \\
\hline Perito Facultativo de Minas & $\begin{array}{l}\text { Ensayadores } \\
\text { Peritos Beneficiadores } \\
\text { Peritos Ingenieros de Minas }\end{array}$ & $\begin{array}{l}\text { Ingeniero de Minas } \\
\text { Ensayador } \\
\text { Topógrafo } \\
\text { Beneficiador de Metales } \\
\text { Apartador }\end{array}$ & $\begin{array}{l}\text { Ingeniero de Minas y Metalurgista } \\
\text { Ensayador y Apartador de Metales } \\
\text { Topógrafo e Hidrógrafo } \\
\text { Ingeniero Geógrafo } \\
\text { Ingeniero Industrial } \\
\text { Ingeniero Civil } \\
\text { Electricista }\end{array}$ \\
\hline
\end{tabular}

Fuente: Ramos-Lara, 2013: 109 y 111.

\footnotetext{
${ }^{11}$ Bazant (1992) ha explicado esta situación con los ingenieros de minas.
} 


\section{Gráfica 1. Comparación del número de alumnos graduados en la ENI: 1890-1900, 1900-1910}

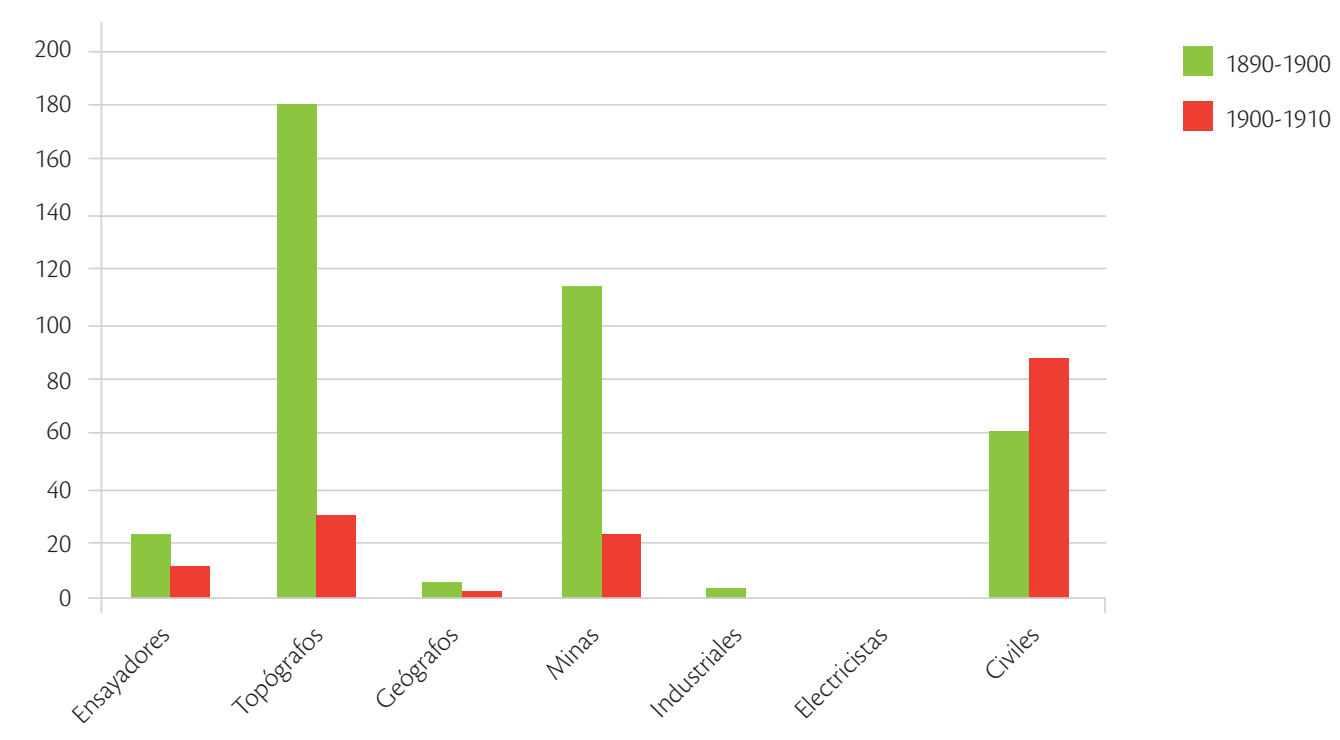

Fuente: Ramos-Lara, 2013: 151.

A principios del siglo XX, el descenso en el número de estudiantes graduados fue alarmante, tanto que las autoridades de la institución reunieron al personal académico de la escuela, a los miembros de la Asociación de Ingenieros Civiles y Arquitectos y a las autoridades del Ministerio de Instrucción Pública para buscar soluciones inmediatas. El diagnóstico del problema señalaba dos causas primordialmente:

a) La exagerada enseñanza teórica más que práctica en la escuela, que impedía que los estudiantes tuvieran la capacidad manual y técnica requerida en las industrias, principalmente de los cursos de física. ${ }^{12}$

b) La falta de demanda laboral en el sector industrial para los jóvenes mexicanos.
El Ministerio de Instrucción Pública se inclinó por la primera explicación y difundió la idea de que los ingenieros mexicanos no eran contratados por las empresas porque no tenían la misma formación que los extranjeros. A diferencia de esa apreciación, actualmente hay trabajos que muestran lo contrario, es decir, los planes de estudios de la ENI en algunas carreras eran más sólidos que algunos en Estados Unidos y en Europa. ${ }^{13} \mathrm{El}$ ejercicio práctico no lo tenían porque las grandes empresas foráneas se negaban a recibir estudiantes mexicanos para realizar prácticas de estudios.

La situación de la ENI se recrudeció a partir de 1910, con el advenimiento de la Revolución mexicana, como se muestra en la gráfica 2.

\footnotetext{
${ }^{12}$ Esto se entiende a la luz de que la industria en esos años había sido producto de importantes avances en la física, como la termodinámica, la electricidad y el magnetismo, además de novedosas formalizaciones de la mecánica.

${ }^{13}$ Ver Bazant (1992) y Ramos-Lara (2013).
} 
Gráfica 2. Matrícula en la ENI, años 1909, 1913, 1914

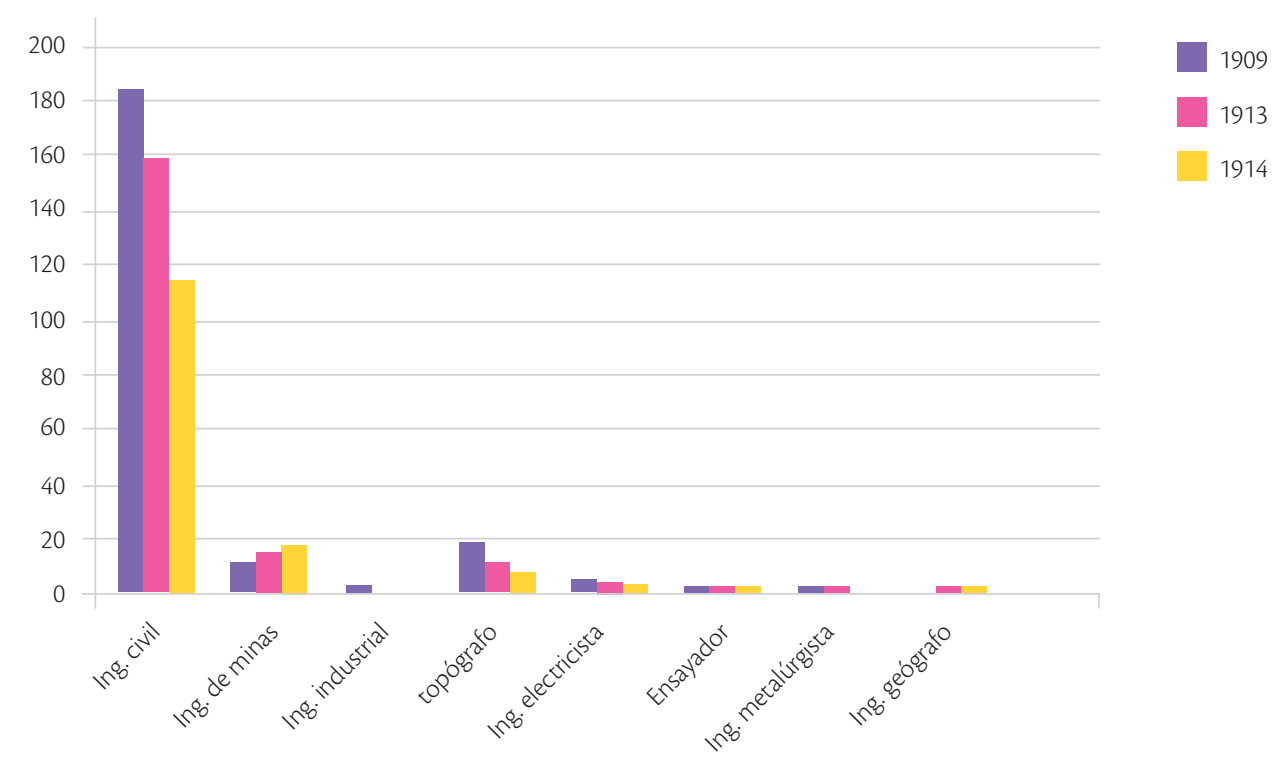

Fuente: Ramos-Lara, 2013: 152.

El fenómeno fue devastador para la ENI, pues se anulaban prácticamente las carreras industriales cuya plataforma científica se fundamentaba en las matemáticas, la física y la química. De este análisis cabe preguntarse si estas disciplinas tendrían mejores oportunidades de desarrollo al crearse la Universidad, y la respuesta evidente es negativa, dado que el sistema económico y los factores externos, en general, eran los mismos.

\section{Creación de la Universidad Nacional de México}

Como mencioné, la Real y Pontificia Universidad fue clausurada en 1865, así que México dejó de tener universidad en la capital del país. Para cubrir este vacío, en 1881, el distinguido abogado y entonces diputado Justo Sierra presentó a la Cámara de Diputados el proyecto de creación de una nueva universidad, el cual estaba estrechamente vinculado con la fundación de una Escuela de Altos Estudios. La respuesta del congreso fue negativa, y se le pidió fortalecer la educación en todos los niveles antes de pretender crear altos estudios en la República mexicana (Ducoing, 1990).

Así, Justo Sierra se dio a la tarea de crear programas educativos que fortalecieran todas las instituciones y la ENI fue de las más beneficiadas, de hecho, pasó de ser Escuela Especial a Escuela Nacional en 1883. Los planes de estudios se renovaron y fortalecieron, la matrícula se incrementó, se crearon nuevas carreras, se aumentó el presupuesto para modernizar el equipo de los laboratorios y actualizar el material de la biblioteca, etcétera. Todo ello la condujo a un gran despunte que se vio reflejado satisfactoriamente en 1886. Todavía continuó su crecimiento durante algunos años cuando sobrevino el colapso de la escuela (Ramos-Lara, 2013). ${ }^{14}$

\footnotetext{
${ }^{14}$ De acuerdo con los sistemas complejos, el colapso de la ENI se explica en términos de que el sistema comenzó a ser vulnerable por los efectos nocivos de los factores externos, y su fragilidad fue tal que una pequeña perturbación tuvo consecuencias desastrosas, de ahí su caída tan precipitada (Ramos-Lara, 2013; García, 1993).
} 
Justo Sierra mantuvo latente su plan académico y lo empezó a gestionar cuando fue nombrado ministro de Instrucción Pública y Bellas Artes (en 1905). Su proyecto se centraba en la creación tanto de la Universidad como de la Escuela Nacional de Altos Estudios (ENAE) (Alvarado, 2013). En realidad, el más original era el segundo, pues el primero sólo consistía en agrupar las escuelas nacionales, que en esos momentos eran las más sólidas y sobresalientes académicamente a nivel nacional.

Finalmente, en 1910 se crearon estas dos instituciones como parte de las suntuosas festividades realizadas en torno al centenario de la Independencia del país. El 18 se septiembre de 1910 se fundó la ENAE y cuatro días más tarde la UNM. La Universidad integró las escuelas nacionales que venían funcionando de manera independiente: la ENI desde 1792, la ENAB desde 1781, la ENM desde 1833, la ENJ y la ENP desde 1867, como se muestra en el diagrama 1 (Ruiz, 1967).
La única escuela de nueva creación fue la ENAE, la joya de Justo Sierra, en la cual se impartirían los estudios científicos, sociales y de humanidades que no había en México, como: física, matemáticas, química, biología, lenguas clásicas, literatura, filología, pedagogía, lenguas vivas, psicología, ética, estética, filosofía e historia de las doctrinas filosóficas, ciencias políticas, sociales y jurídicas (Menéndez, 1996). Además de estar encargada de realizar investigación científica de frontera, para lo cual había quedado como el organismo dedicado a organizar las instituciones científicas de la época. De igual manera sería una institución destinada a formar profesores en diversos campos y niveles.

En el proyecto inicial, las ciencias físicas tenían una presencia mayor que las demás, como se evidencia en la gráfica 3, donde se muestra el número de cursos propuestos en 1911 y 1922 en la ENAE para las secciones de ciencias exactas y humanidades.

\section{Diagrama 1. El sistema preparatoriano en 1867 y el sistema universitario de la UNM en 1910}

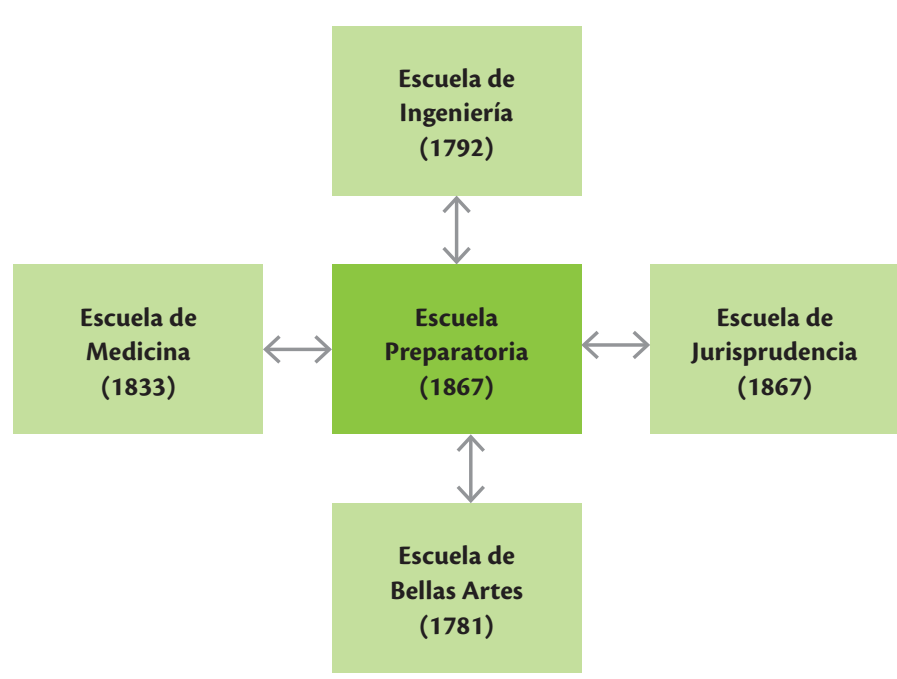

Fuente: Ramos-Lara, 2018: 101.

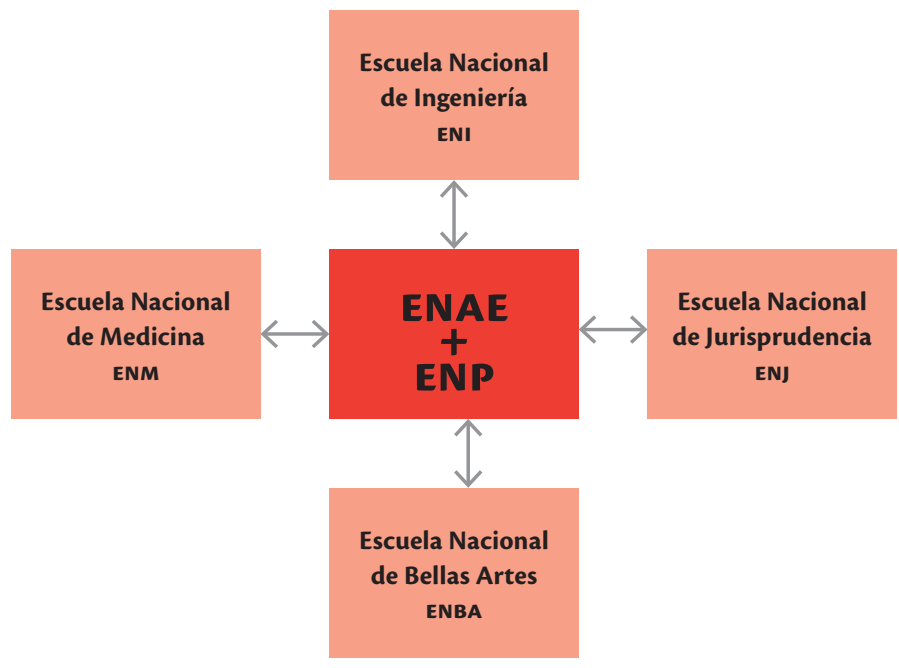

Universidad Nacional Autónoma de México 


\section{Gráfica 3. Materias programadas en la ENAE en 1911 y 1922}

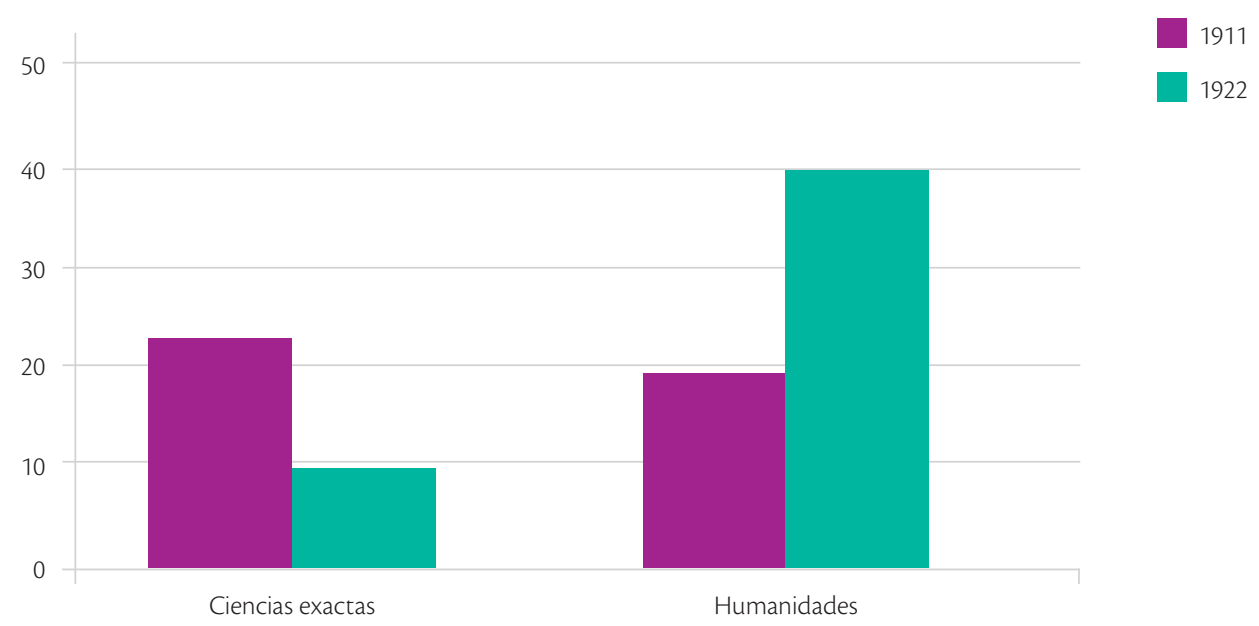

En dicha gráfica se observa cómo en los programas de estudios iniciales (1911) se favorecía a las ciencias físicas además de que se aprovechaban las instalaciones de las escuelas nacionales y de las instituciones científicas para que las cátedras pudieran desarrollarse plenamente. Nuevamente hubo factores que incidieron negativamente en su desempeño, afectando drásticamente la matrícula de algunos cursos, e imposibilitando en muchos casos su apertura. De esa forma, las humanidades fueron las únicas que lograron estructurar planes de estudios por áreas, como historia, filosofía y letras, de tal manera que en 1924 se decidió transformar la ENAE en Facultad de Filosofía y Letras de la UNM.

La física y las matemáticas quedaban relegadas nuevamente. La química tuvo una suerte diferente, ya que un joven mexicano estudió química en Europa a principios del siglo XX y a su regreso inauguró la Escuela Nacional de Química Industrial en 1916, la cual dependía directamente del Ministerio de Instrucción Pública. Los factores externos no tardaron en perjudicarla, pues meses después de su fundación fue clausurado ese Ministerio durante el movimiento revolucionario. Ante ese panorama, su director, Juan Salvador Agraz, solicitó su incorporación a la UNM, hecho que tuvo lugar el 5 de febrero de 1917, bajo la denominación de Facultad de Ciencias Químicas (León, 2014).

La biología también pudo desarrollarse en la década de los veinte en la misma ENAE, donde se promovieron los estudios de maestría y doctorado (Hoffmann et al., 1993). Sólo la física y las matemáticas, los campos fundamentales de la ingeniería y por ende de la industria, seguían relegados. Fue hasta 1935 que el ingeniero civil egresado de la ENI, Ricardo Monges López, decidió crear el Departamento de Ciencias Físicas y Matemáticas con el apoyo de la ENI - como era evidente - para iniciar los estudios profesionales de dichas disciplinas. $\mathrm{Al}$ año siguiente, el departamento fue transformado en Escuela Nacional de Ciencias Físicas y Matemáticas, y, finalmente, en 1938 cambió a Facultad de Ciencias, nombre que continúa hasta hoy (Ramos-Lara, 2015). 


\section{Conclusiones}

La idea de profesionalizar disciplinas científicas y humanísticas, además de desarrollar investigación científica de frontera en México, fue parte de los objetivos de la creación de la Universidad Nacional de México en 1910, pero por la influencia negativa de los factores externos (las empresas extranjeras que se instalaron en este país y las diferencias con el sector gubernamental, entre otras) el proyecto científico decayó, por lo que el nacimiento de algunos campos, como el de la química, tuvieron que iniciar fuera de la universidad, en tanto que los de física y matemáticas quedaron en estado decadente por casi 25 años, hasta que tuvo lugar una reorganización política del país, producto de gobiernos

\section{Referencias}

Alvarado, Lourdes (comp.) (2013), El proceso de la creación de la Universidad Nacional de México a través de las fuentes documentales, México, UNAM/Siglo XXI.

Bazant, Mílada (1992), "La enseñanza y la práctica de la ingeniería durante el porfiriato", en La educación en la historia de México, México, El Colegio de México, pp. 167-210.

Bertalanffy, Ludwig Von (1968), Teoría General de los Sistemas, México, Fondo de Cultura Económica.

Brading, David (1993), Mineros y comerciantes en el México borbónico (1763-1810), México, Fondo de Cultura Económica.

Cosío Villegas, Daniel (1963), "El porfiriato, era de consolidación", en Historia Mexicana, vol. 13, no. 1, julio-septiembre, pp. 76-87.

De Gortari, Eli (1980), La ciencia en la historia de México, México, Editorial Grijalbo.

Domínguez, José Raúl (2013), La ingeniería civil en México 1900-1940. Análisis histórico de los factores de su desarrollo, México, UNAM.

Ducoing, Patricia (1990), La pedagogía en la Universidad de México 1881-1954, t. I, México, UNAM. posrevolucionarios, y que venía a la par de diversos conflictos al interior de la UNM que demandaban su autonomía. En 1929, la Universidad conquistó su autonomía e inició un proceso de auto-organización interna que le facilitó la delimitación de sus fronteras con el aparato gubernamental (factor externo), propiciando condiciones favorables para la creación de profesiones en ciencias físicas y matemáticas, y de instituciones de investigación para el desarrollo de la ciencia básica y de frontera, en una amplia gama de campos. Como consecuencia de este escenario, se predispuso una barrera entre la producción del conocimiento al interior de la Universidad y las necesidades del tan vulnerado sector industrial mexicano.

Gallardo, Juan Carlos, Juan Manuel Lozano y María de la Paz Ramos-Lara (2005), "Publicaciones sobre temas de física en las Memorias de la Sociedad Científica Antonio Alzate", Ciencia Ergo Sum, 12(1), pp. 97-104.

García, Rolando (1993), "Teorías de sistemas y ciencias sociales", en Ignacio Méndez y Pablo González Casanova (coords), Matemáticas y ciencias sociales, México, UNAM/Miguel Ángel Porrúa, pp. 89-116.

García, Rolando (2006), Sistemas complejos, Barcelona, Editorial Gedisa.

Hoffmann, Anita, José Luis Cifuentes y Jorge Llorente (1993), Historia del Departamento de Biología de la Facultad de Ciencias UNAM, México, UNAM.

Izquierdo, José Joaquín (1958), La primera casa de las ciencias en México. El Real Seminario de Minería (1792-1811), México, Ediciones Ciencia.

Lassaga, Juan Lucas y Joaquín Velázquez Cárdenas de León (1774), Representación que a nombre de la Minería de esta Nueva España, hacen al Rey Nuestro Señor los apoderados de ella, D. Juan Lucas de Lassága, Regidor de esta Nobilísima Ciudad, y Juez Contador de Menores, y Albaceazgos: y D. Joaquin Velasquez de Leon, Abogado de esta Real Audiencia, y 
Catedrático que ha sido de Matemáticas en esta Real Universidad, México, Imprenta Felipe de Zúñiga y Ontiveros.

León, Felipe (2014), "Génesis de la formación de químicos en México", en Aportes recientes a la historia de la química en México, México, UNAM, pp. 187-200.

Martínez, Gustavo (2012), "Sistemas complejos: entrevista con el Dr. Gustavo Martínez Mekler”, Revista Digital Universitaria, vol. 13, núm. 4.

Méndez Arceo, Sergio (1990), La Realy Pontificia Universidad de México, México, UNAM.

Menéndez, Libertad (1996), "Escuela Nacional de Altos Estudios y Facultad de Filosofía y Letras. Planes de estudios, títulos y grados 1910-1994", Tesis de Doctorado en Pedagogía, México, UNAM.

Miramontes, Pedro (1999 a), "El estructuralismo dinámico", en Santiago Ramírez (coord.), Perspectivas en las teorías de sistemas, UNAM/Siglo XXI Editores, pp. 70-82.

Miramontes, Octavio (1999 b), "Los sistemas complejos como instrumentos de conocimiento y transformación del mundo", en Santiago Ramírez (coord.), Perspectivas en las teorías de sistemas, UNAM/Siglo XXI Editores, pp. 83-92.

Ramos-Lara, María de la Paz (2018), La Escuela Nacional Preparatoria. Un sistema complejo adaptativo, México, UNAM.
Ramos-Lara, María de la Paz (2015), "En los 75 años de la Facultad de Ciencias, semblanza de su fundador Ricardo Monges", en Ciencias, 115-116, enero-junio 2015, pp. 140-149.

Ramos-Lara, María de la Paz (2014), "La química en los inicios de la Escuela Nacional de Altos Estudios", en Aportes recientes a la historia de la química en México, México, UNAM, pp. 173-186.

Ramos-Lara, María de la Paz (2013), Vicisitudes de la ingeniería en México (siglo XIX), México, UNAM.

Ruiz, Beatriz, (1967), "Justo Sierra y la Escuela de Altos Estudios", en Historia Mexicana, México, vol. 16, núm. 4 (64), pp. 541-564.

\section{Fuentes primarias de consulta general}

Archivo General de la Nación

Archivo Histórico de la Universidad Nacional Autónoma de México (AHUNAM), Fondos:

1. Escuela Nacional de Ingenieros

2. Escuela Nacional de Altos Estudios

3. Escuela Nacional de Ciencias Químicas

Archivo Histórico del Palacio de Minería de la Facultad de Ingeniería

\section{Cómo citar este artículo:}

Ramos-Lara, María de la Paz (2019), “Condiciones adversas para la ciencia al nacer la Universidad Nacional de México", en Revista Iberoamericana de Educación Superior (RIES), México, UNAM-IISUE/Universia, vol. x, Núm. 28, pp. 83-96, DOI: https://doi.org/10.22201/ iisue.20072872e.2019.28.430 [consulta: fecha de última consulta]. 